\title{
Sterile cockpit or not: It's all about team and effective communication
}

\author{
David J. Rosinski, MPS, LCP
}

In the February 2010 issue of this Journal, an article appeared discussing the impact of protocol-driven communication during cardiopulmonary bypass. ${ }^{1}$ As the authors pointed out in their summary, the cardiac surgical operating room is an intense environment with significant complexity and a cadre of well-meaning, continuously multitasking individuals. Although the check and balance system designed for patient safety has proved its merit through the years, there is a difference in terms of effective reactive communication versus proactive communication for a surgical team. Therefore the "sterile cockpit"' philosophy, although useful may not be broadly applicable to critical time rather than critical events. Of interest was the discussion after the article. In response to a question related to breakdowns in communication between surgeons and perfusionists, Dr Sundt relayed a recent experience involving activated clotting time management. As a perfusionist, I was pleased to read Dr Sundt's reply, because it presented a reality that many of my peers have been concerned with for some time. As Dr Sundt digressed into their change in heparin administration protocol, it was recounted that after protocol change, initiation of cardiopulmonary bypass without even the administration of heparin had almost occurred several times. This being concern enough, what I found even more alarming was the perfusion response: "Well, Dr So-and-So goes on CPB without asking what the ACT is.' 'Further, Dr Sundt went on to say after questioning such perfusion conduct, "The perfusionists are not personally at fault-this is the culture in our institution.' That may be so, yet I find the perfusion response old, tired, an attempt to shift responsibility or defer responsibility, and not contemporaneous with what perfusion practice in 2010 should be. Likewise, I have experienced a situation when an eager young medical student was observing for the first time an open cardiac surgical procedure. With eyes wide, he asked the surgeon during cannulation "Is that the heart-lung machine behind you?" only to hear the following: "Mr Rosinski will tell you that it is extracorporeal circulation with artificial oxygenation involving systemic anticoagulation, myocardial arrest and reperfusion. But it's just a pump.' Clearly more than operating a pump is occurring during bypass. There

\footnotetext{
From the University of Connecticut Health Center, Farmington, Conn, the Heart Center of Greater Waterbury, Waterbury, Conn, and Quinnipiac University Perfusion Education Program, Hamden, Conn.

Received for publication March 5, 2010; accepted for publication March 13, 2010. Address for reprints: David J. Rosinski, MPS, LCP, University of Connecticut Health Center, Cardiac Surgery, 263 Farmington Ave, Farmington, CT 06030 (E-mail: Drosinski@uchc.edu).

J Thorac Cardiovasc Surg 2010;140:10-1

$0022-5223 / \$ 36.00$

Copyright (c) 2010 by The American Association for Thoracic Surgery doi:10.1016/j.jtcvs.2010.03.016
}

has been a long, strong history of perfusion as a profession in the successful evolution of cardiac surgery. To date, the technology, practice, and conduct of cardiopulmonary bypass results in safe, acceptable, patient experience, yet we remain always on the cusp of injury, accident, or poor outcome as patients are seen with more and significantly advanced comorbid conditions. Advanced perfusion technology, along with greater individual or technology-related responsibilities, can overwhelm an individual perfusionist. Effective, proactive communication is therefore paramount. This example is concerning for the following reasons: Are we simply to conduct cardiopulmonary bypass as Dr So-and-So requests? Are we not to interact as a vested team member in each individual patient's best interest? Are we not involved enough that a change in anticoagulation administration protocol can occur without perfusion involvement? Does the role of a perfusionist exist in a vacuum, whereby effective communication with the surgical team fails to occur from within perfusion as a department or allied health profession?

For some time, there has been a call from within our profession to step up and raise our level of play. This example demonstrates the work we need to do. I would consider the response to Dr Sundt unacceptable. There has evolved a chasm within perfusion, resulting in a pattern that contributes to a significant gap between knowledge and practice. Uninvolved perfusionists (those who safely and competently manage patients every day on bypass but do not contribute to an integrated, team-focused, programmatic approach to cardiac surgery) help to enable what the authors described as communication failures: "Despite their negative impact on the work environment, such failures may remain unresolved, because staff members often use process work-arounds that may solve immediate challenges but do not address longterm systemic inadequacies.' As perfusionists, we must actively and aggressively pursue effective communication within the cardiac surgical arena despite common experiences whereby a group of surgeons may refer to their perfusion colleagues as "pump techs" - a perspective that we may bring upon ourselves. Being involved with policies, care plans, outcomes analysis, and case reviews-and continuously striving to advance our practice-will foster a collegial environment preventing such a calamity as starting bypass without heparin administration. So much so that with the ever-increasing complexity of what we do for our surgical colleagues (minimally invasive procedures, hybrid procedures, reservoirless closed minibypass circuits, transfusion avoidance, improved safety and quality, Society of Thoracic Surgeons data management, and so on), proactive communication must come first from patient admission to 
discharge. The power of effective communication can serve to synergize a collaborative approach at the individual patient level (allowable nadir hematocrit, management of coagulopathy, temperature, neurologic protection, or any of a host of physiologic and pathophysiologic conditions that we encounter) as well as to support program changes such as anticoagulation protocol revision, protocol dissemination, and monitoring of the revised protocol's effect. At the very least, effective communication must take place within each operating room among surgeon, anesthesiologist, perfusionist, and nurse patient by individual patient, whereby collectively as a team we all contribute to the patient's best possible experience.

In December 2007, The New Yorker published an article revealing the power of the work being conducted by Dr Peter Pronovost regarding checklist use in medicine with the title "The Checklist: If Something So Simple Can Transform Intensive Care, What Else Can It Do?", ${ }^{2}$ Now, 3 years later, we are beginning to see the fruits of work being done specifically related to safety in cardiac surgery spearheaded by the Society of Cardiac Anesthesia called FOCUS (Flawless Operative Cardiovascular Unified Systems). The FOCUS initiative is multidisciplinary in nature and incorporates collaborative team approaches among anesthesiologist, surgeons, perfusionist, and nurses. In his inaugural editorial for this Journal, Dr Larry Cohn revealed to us his vision not only for the Journal but for cardiovascular surgery as well. With an increasing level of complexity of medical technology and surgical procedures there is "a growing awareness of the interrelationship of our specialty with cardiovascular and thoracic anesthesiologists, cardiologists, pulmonologists, intensivists, and cardiopulmonary bypass perfusionists, as well as basic scientists., ${ }^{3}$ As of 2010 , with all of us on the same team, working toward like goals with equally good intentions, it is completely acceptable to hold all of us accountable and responsible for the realization of a high-quality standard of care. Perfusionists do not need to hide behind Dr So-and-So, and surgeons do not need to hide behind being a physician. I appreciate the work conducted by Dr Sundt and his team. The importance of effective communication in quality patient care is as relevant today as it was during the initial days of cardiopulmonary bypass-supported intracardiac surgery.

\section{References}

1. Wadhera RK, Parker SH, Burkhart HM, Greason KL, Neal JR, Levenick KM, et al. Is the "sterile cockpit"' concept applicable to cardiovascular surgery critical intervals or critical events? The impact of protocol-driven communication during cardiopulmonary bypass. J Thorac Cardiovasc Surg. 2010;139:312-9.

2. Gawande A. The checklist: if something so simple can transform intensive care, what else can it do? New Yorker. 2007 Dec 10;86-101.

3. Cohn LH. What is changing and what isn't in The Journal of Thoracic and Cardiovascular Surgery: the cardiovascular and thoracic service-line concept. $J$ Thorac Cardiovasc Surg. 2008;135:1-2. 